Silvia Elaine Cardozo Macedo'

Ana Maria Baptista Menezes'

Elaine Albernaz"

Paulo Post "II

Marli Knorst ${ }^{\text {IV }}$
Departamento de Clínica Médica.

Universidade Federal de Pelotas (UFPel).

Pelotas, RS, Brasil

Departamento de Pediatria. Universidade Católica de Pelotas. Pelotas, RS, Brasil

III Departamento de Microbiologia. UFPel. Pelotas, RS, Brasi

IV Departamento de Medicina Interna. Universidade Federal do Rio Grande do Sul. Porto Alegre, RS, Brasil

Correspondência | Correspondence: Silvia Elaine Cardozo Macedo

Rua Dr. José Bonifácio de Carvalho Costa, 387

96020430 Pelotas, RS, Brasil

E-mail: secmacedo@terra.com.br

\section{Fatores de risco para internação por doença respiratória aguda em crianças até um ano de idade}

\section{Risk factors for acute respiratory disease hospitalization in children under one year of age}

\section{RESUMO}

OBJETIVO: Avaliar fatores de risco para hospitalização por doença respiratória aguda em crianças até um ano de idade.

MÉTODOS: Estudo de casos e controles na cidade de Pelotas, RS. Os casos foram crianças de até um ano de idade, que se hospitalizaram por doença respiratória aguda, de agosto de 1997 a julho de 1998. Os controles foram crianças da comunidade, da mesma idade, sem hospitalização prévia por essa doença. Um questionário investigando exposição a fatores de risco foi aplicado às mães de casos e controles. Os dados foram submetidos à análise univariada, bivariada e multivariada por meio de regressão logística para avaliação dos fatores de risco sobre o desfecho de interesse.

RESULTADOS: Foram analisadas 777 crianças, sendo 625 casos e 152 controles. $\mathrm{Na}$ análise bruta, os fatores de risco associados ao desfecho foram: sexo masculino, faixa etária menor de seis meses, aglomeração familiar, escolaridade materna, renda familiar, condições habitacionais inadequadas, desmame precoce, tabagismo materno, uso de bico, história de hospitalização e antecedentes de sintomas respiratórios. O trabalho materno foi fator de proteção para internação por doença respiratória aguda. Na análise multivariada, permaneceram associadas: ausência de ou baixa escolaridade materna $(\mathrm{OR}=12,5)$, história pregressa de sibilância $(\mathrm{OR}=7,7)$, desmame precoce $(\mathrm{OR}=2,3)$, uso de bico $(\mathrm{OR}=1,9)$, mãe fumante $(\mathrm{OR}=1,7)$, idade abaixo de seis meses $(\mathrm{OR}=1,7)$ e sexo masculino $(\mathrm{OR}=1,5)$.

CONCLUSÕES: Os resultados mostraram a importância dos aspectos sociais e comportamentais da família, assim como morbidade respiratória anterior da criança como fatores de risco para hospitalização por doença respiratória aguda.

DESCRITORES: Infecções respiratórias. Fatores de risco. Estudos de casos e controles. 


\section{ABSTRACT}

OBJECTIVE: To evaluate risk factors for acute respiratory disease hospitalizations in children under one year of age.

METHODS: A case-control study was conducted in the city of Pelotas, Southern Brazil. Cases were children under one year of age who were hospitalized due to acute respiratory diseases from August 1997 to July 1998. Controls were same-age community children randomly selected without previous respiratory disease hospitalization. A questionnaire about risk factors exposure was applied to the mothers of cases and controls. Univariate, bivariate and multivariate analyses through logistic regression were carried out to evaluate risk factors for the outcome of interest.

RESULTS: There were studied 777 children; 625 cases and 152 controls. In the crude analysis, the risk factors associated with the outcome were: being male, children under six months of age, household crowding, maternal education, family income, inadequate housing conditions, lack of breastfeeding, maternal smoking, use of pacifiers, and a previous history of hospitalization and respiratory symptoms. Maternal working was a protection factor associated with acute respiratory disease hospitalizations. In the multivariate analysis the following risk factors remained associated: maternal education $(\mathrm{OR}=12.5)$, previous history of wheezing $(\mathrm{OR}=7.7)$, lack of breastfeeding $(\mathrm{OR}=2.3)$, use of pacifiers $(\mathrm{OR}=1.9)$, maternal smoking $(\mathrm{OR}=1.7)$, children under six months of age $(\mathrm{OR}=1.7)$, and being male $(\mathrm{OR}=1.5)$.

CONCLUSIONS: The study results show the importance of the family's social and behavioural aspects as well as previous respiratory disease as risk factors for acute respiratory disease hospitalizations in children under one year of age.

KEYWORDS: Respiratory tract infections. Risk factors. Case-control studies.

\section{INTRODUÇÃO}

As doenças respiratórias agudas (DRA) e, particularmente as infecções respiratórias agudas (IRA), são uma das causas mais comuns de morbimortalidade na infância, atingindo principalmente crianças menores de cinco anos de idade. Apesar do decréscimo observado na mortalidade por IRA, tanto em países desenvolvidos como naqueles em desenvolvimento, a redução foi muito maior entre os desenvolvidos, e a morbidade permanece significativa em ambos. ${ }^{6}$

No Rio Grande do Sul, cerca de 30,4\% das consultas pediátricas nos meses de inverno são motivadas por DRA. Similarmente, a análise das causas que contribuem para a mortalidade na infância mostra que logo após as afecções originárias no período perinatal, as pneumonias constituem-se no principal grupo. Elas perfazem cerca de $11,6 \%$ do total de mortes na faixa etária menor de um ano, e 16,1\% na faixa etária de um a quatro anos de idade. ${ }^{5}$
A magnitude do problema, especialmente nos países em desenvolvimento, apontam a necessidade de busca constante de estratégias efetivas para o controle da situação, como o reconhecimento e intervenção sobre seus fatores de risco.

Em relação aos fatores de risco para DRA, são encontrados na literatura resultados algumas vezes controversos, com risco observado apenas para determinados grupos de DRA, ou para desfecho clínico específico, como a mortalidade sem efeito sobre a morbidade por DRA. Em linhas gerais, no entanto, o baixo nível socioeconômico, ${ }^{15}$ as condições ambientais inadequadas, incluindo aglomeração familiar ${ }^{14} \mathrm{e}$ exposição passiva ao fumo, ${ }^{4,14}$ são apontados como os principais fatores de risco envolvidos na ocorrência das DRA na infância. Além desses aspectos, há aqueles relacionados às condições nutricionais (baixo peso ao nascer, baixos peso e estatura para a idade e interrupção da amamentação) $)^{13} \mathrm{e}$ a presença de doenças respiratórias pré-existentes na criança. ${ }^{13}$ 
Em algumas situações, é difícil distinguir as condições respiratórias agudas entre lactentes. Freqüentemente, na prática pediátrica, há dificuldade de discriminar quadros de infecções de vias aéreas inferiores de crises asmáticas. Por outro lado, não é incomum, que os quadros infecciosos de vias aéreas alta ou baixa, especialmente os virais, sejam responsáveis por exacerbações de asma. Dessa forma, observa-se na literatura uma variedade de expressões na definição das condições respiratórias agudas em lactentes. Expressões como "doença respiratória baixa", "doença do trato respiratório inferior" ou "doença respiratória baixa sibilante", 12 "doença respiratória aguda baixa" " são vistas com certa freqüência. Essas expressões também denotam a dificuldade de separar a asma de outras condições respiratórias agudas, incluindo quadros infecciosos, que cursam nesse grupo etário com sibilância. Em artigo anterior, ${ }^{11}$ avaliou-se a incidência de internações por quadros respiratórios agudos em lactentes e para o presente estudo, definiu-se o desfecho investigado como DRA, incluindo infecções de vias aéreas superiores e inferiores, pneumonias e crises asmáticas.

O presente estudo teve por objetivo avaliar fatores de risco para hospitalização por doença respiratória aguda de crianças até um ano de idade.

\section{MÉTODOS}

Realizou-se um estudo de caso-controle nos quatro hospitais da cidade de Pelotas, RS, no período de 1 de agosto de 1997 a 31 de julho de 1998. Os casos foram representados por crianças hospitalizadas (UTI pediátricas e enfermarias), de zero a um ano de idade, com diagnóstico de DRA na baixa hospitalar. Posteriormente, um árbitro independente (médico pediatra) revisava as informações sobre o caso e, baseando-se nos critérios propostos pela Organização Mundial de Saúde* (OMS), decidia o diagnóstico da criança. As crianças que tiveram outros diagnósticos, que não de DRA, pelo árbitro, foram excluídas do estudo. Os controles foram crianças aleatoriamente selecionadas da comunidade com até um ano de idade, que não haviam sido hospitalizadas por DRA até aquele momento. Para a seleção aleatória dessas crianças, sortearam-se 20 setores censitários da cidade para encontrar o máximo de oito crianças por setor. No entanto, não foi encontrado esse número em sete setores, procedendo-se sorteio adicional de outros quatro setores. Em cada setor selecionado, era sorteado o quarteirão inicial e, posteriormente uma esquina por onde o entrevistador deveria começar a busca. A partir desse ponto, todos os domicílios eram visitados até encontrar uma criança que preenchesse os critérios para ser um controle. A busca dos casos ocorreu por meio de visitas diárias aos hospitais da cidade, onde as crianças com diagnóstico na baixa hospitalar de DRA eram identificadas. A mãe ou responsável pela criança respondia a um questionário estruturado e pré-codificado, contendo questões referentes às condições sociais, de nascimento e história médica pregressa da criança (antecedentes de sintomas respiratórios, hospitalizações anteriores, e outros). O mesmo questionário foi também aplicado às mães das crianças controles.

No período do estudo ocorreram 874 internações consideradas casos. As recusas e perdas atingiram 8,8\% da amostra $(\mathrm{N}=57)$, resultando em 817 internações. Deste total, foram excluídos 81 casos por: ausência de diagnóstico de alta hospitalar no prontuário médico, dados insuficientes para a realização de diagnóstico adequado pelo árbitro do estudo, ou porque o diagnóstico do árbitro não era de DRA. O total de casos

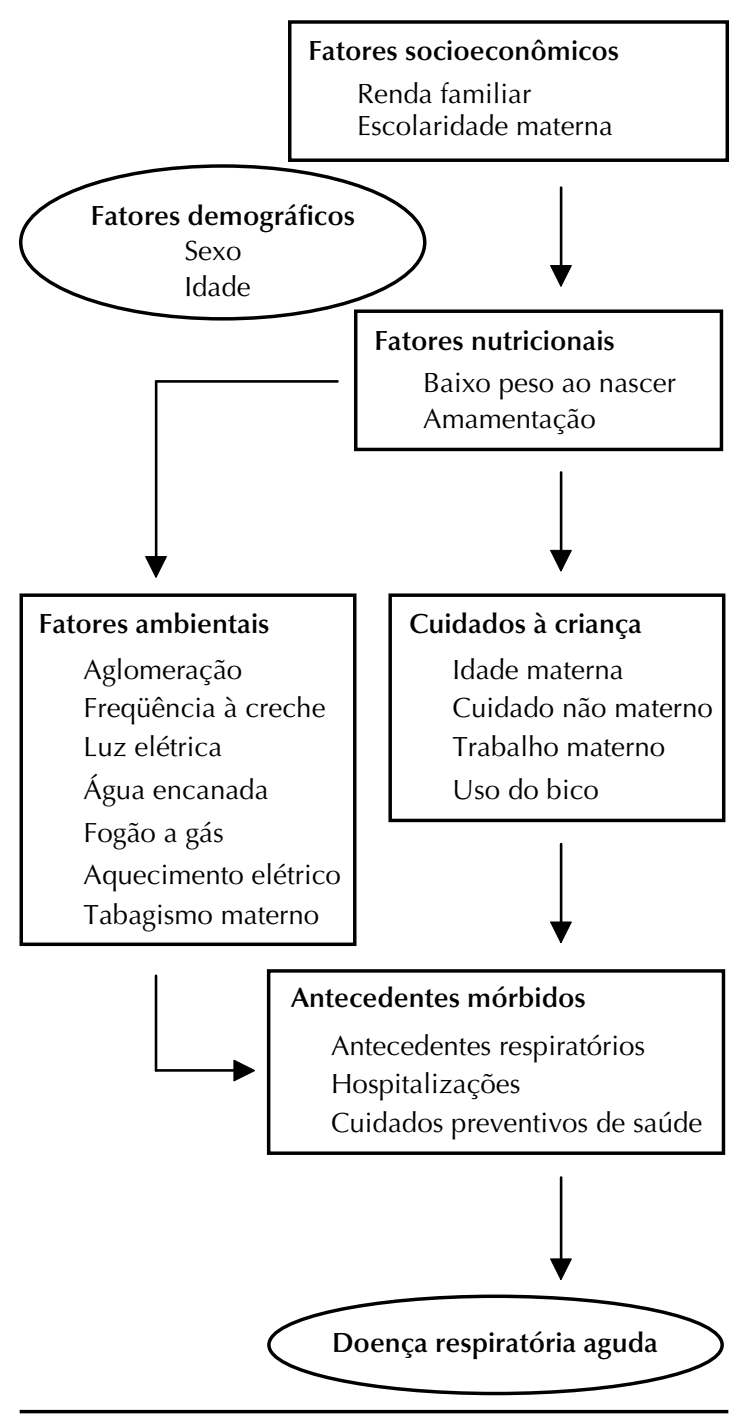

$\overline{\text { Figura. Modelo teórico hierarquizado para determinação de }}$ doença respiratória aguda.

\footnotetext{
* Ministério da Saúde. Divisão Nacional de Epidemiologia. Manual de normas para infecção respiratória aguda, Brasília;1991.
} 
Tabela 1. Comparação de casos de hospitalização por doença respiratória aguda e controles, em análise bivariada, quanto às características demográficas, socioeconômicas e maternas. Pelotas, RS, 1998.

\begin{tabular}{|c|c|c|c|c|c|}
\hline Variável & $\begin{array}{l}\text { Casos } \\
\mathrm{N}(\%)\end{array}$ & $\begin{array}{c}\text { Controles } \\
\mathrm{N}(\%)\end{array}$ & OR & IC $95 \%$ & $\mathrm{p}$ \\
\hline \multicolumn{6}{|l|}{ Sexo } \\
\hline Feminino & $264(42,2)$ & $81(57,8)$ & 1,0 & & \\
\hline Masculino & $361(53,3)$ & $71(46,7)$ & 1,5 & 1,$1 ; 2,2$ & 0,01 \\
\hline \multicolumn{6}{|l|}{ Faixa etária ( meses) } \\
\hline 1 a 6 & $413(66,1)$ & $80(52,6)$ & 1,7 & 1,$2 ; 2,5$ & $<0,01$ \\
\hline 6,1 a 12 & $212(33,9)$ & $72(47,4)$ & 1,0 & & \\
\hline \multicolumn{6}{|c|}{ No de pessoas que dormem com a criança } \\
\hline Até 3 & $402(65,0)$ & $115(79,3)$ & 1,0 & & \\
\hline$>$ de 3 & $216(35,0)$ & $30(20,7)$ & 2,1 & 1,$3 ; 3,2$ & $<0,01$ \\
\hline \multicolumn{6}{|l|}{ Escolaridade materna (anos) } \\
\hline$\geq 9$ & $68(10,9)$ & $58(40,0)$ & 1,0 & & \\
\hline 5 a 8 & $319(51,3)$ & $67(46,2)$ & 4,0 & 2,$6 ; 6,2$ & $<0,001$ \\
\hline 1 a 4 & $197(31,7)$ & $18(12,4)$ & 9,2 & 5,$1 ; 16,6$ & $<0,001$ \\
\hline Sem escolaridade & $38(6,1)$ & $2(1,4)$ & 16,7 & 3,$8 ; 74,0$ & $<0,001$ \\
\hline \multicolumn{6}{|l|}{ Renda familiar mensal (SM) } \\
\hline$>6$ & $38(6,2)$ & $23(15,9)$ & 1,0 & & \\
\hline 3,1 a 6 & $109(17,8)$ & $44(30,3)$ & 1,5 & 0,$8 ; 2,8$ & 0,2 \\
\hline 1,1 a 3 & $229(37,4)$ & $51(35,2)$ & 2,7 & 1,$5 ; 4,9$ & $<0,01$ \\
\hline Até 1 & $236(38,6)$ & $27(18,6)$ & 5,2 & 2,$7 ; 9,9$ & $<0,001$ \\
\hline \multicolumn{6}{|l|}{ Não trabalha fora de casa } \\
\hline Não & $497(79,5)$ & $98(66,7)$ & 1,0 & & \\
\hline Sim & $128(20,5)$ & $49(33,3)$ & 0,5 & 0,$3 ; 0,8$ & $<0,01$ \\
\hline \multicolumn{6}{|l|}{ Fumo materno (no cig./dia) } \\
\hline Não fuma & $341(54,6)$ & $110(72,4)$ & 1,0 & & \\
\hline Até 10 & $235(37,6)$ & $39(25,7)$ & 1,9 & 1,$3 ; 2,9$ & $<0,01$ \\
\hline$>10$ & $49(7,8)$ & $3(2,0)$ & 5,1 & 1,$6 ; 16,5$ & $<0,01$ \\
\hline \multicolumn{6}{|l|}{ Amamentação (meses) } \\
\hline Mais de 6 ou ainda mama & $209(33,4)$ & $74(48,7)$ & 1,0 & & \\
\hline $3,1-6$ & $49(7,8)$ & $16(10,5)$ & 1,1 & 0,$6 ; 2,1$ & 0,7 \\
\hline $1,1-3$ & $123(19,7)$ & $23(15,1)$ & 1,9 & 1,$1 ; 3,1$ & 0,02 \\
\hline Até 1 & $244(39,0)$ & $39(25,7)$ & 2,2 & 1,$4 ; 3,4$ & $<0,001$ \\
\hline
\end{tabular}

SM: salário mínimo

atingido foi de 736, das quais 579 tiveram uma única internação e 157 foram novamente internadas por DRA, pelo menos uma vez. Nesse grupo de crianças que tiveram re-internação por DRA, analisaram-se os dados referentes apenas à primeira internação, o que correspondeu a 71 casos, totalizando 650 internações. Em relação às crianças controles calculou-se um tamanho amostral de 146 crianças, acrescendo-se 10\% para eventuais perdas e recusas, o que totalizou 160 crianças. Posteriormente, optou-se por retirar crianças com menos de um mês de vida para anular a influência das afecções perinatais sobre o desfecho, totalizando 625 casos e 152 controles.
Os dados foram submetidos à análise univariada, bivariada e multivariada por meio de regressão logística para avaliação dos fatores de risco sobre o desfecho de interesse. Na análise multivariada (Figura) construiu-se um modelo teórico hierarquizado, o qual contemplou de forma seqüencial com base em conceitos teóricos "a priori” definidos, os diversos fatores de risco envolvidos na cadeia biológica do desfecho de interesse. ${ }^{16}$ Assim, as variáveis de um determinado nível foram controladas pelas variáveis de mesmo nível e de níveis precedentes. Os fatores socioeconômicos foram situados no primeiro nível hierárquico, pois poderiam influenciar todos os demais fatores proximais ao desfecho. A medida de 
efeito utilizada foi o odds ratio (OR) e a significância estatística das associações foi calculada pelo teste de qui-quadrado de Pearson.

O projeto foi aprovado pelo Comitê de Ética do Hospital Escola da Fundação de Apoio Universitário da Faculdade de Medicina de Pelotas. Para a coleta dos dados, solicitou-se o consentimento informado verbal das mães, com a garantia do absoluto sigilo das informações.

\section{RESULTADOS}

Em relação às características demográficas, houve predomínio do sexo masculino na amostra em geral (55,6\%). A média de idade e desvio-padrão dos casos e dos controles foram, respectivamente, de 5,3 $\pm 3,0$ meses, e de 6,2 $\pm 3,2$ meses; $64,9 \%$ do total da amostra apresentava menos de seis meses de idade. Quanto às características socioeconômicas, a renda familiar média

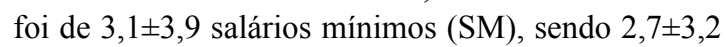
SM entre os casos, e 4,6 $\pm 5,7 \mathrm{SM}$ entre os controles. Cerca de $35 \%$ da amostra possuía rendimento mensal inferior a um salário mínimo.

As causas mais freqüentes de hospitalizações, conforme o árbitro do estudo, foram: pneumonia $(43,7 \%)$, bronquiolite $(31,0 \%)$, asma $(20,3 \%)$, gripe $(3,5 \%)$, otite média aguda $(0,8 \%)$ e laringite $(0,6 \%)$.

As Tabelas 1 e 2 revelam a comparação de casos e controles quanto às características de interesse, na análise bivariada. Associaram-se ao desfecho ser do sexo masculino, faixa etária menor de seis meses, aglomeração familiar, escolaridade materna, renda familiar, condições habitacionais inadequadas, desmame precoce, tabagismo materno, uso de bico, história de hospitalização e antecedentes de sintomas respiratórios. O fato de a mãe trabalhar fora foi fator de proteção para internação por DRA. Não houve associação desse desfecho com a idade materna, baixo peso ao nascimento e freqüência à creche.

A Tabela 3 descreve a comparação de casos e controles, após análise multivariada por regressão logística, em relação aos fatores de risco. Permaneceram associadas à hospitalização por DRA, em ordem decrescente de OR, as seguintes variáveis: ausência de ou baixa escolaridade materna $(\mathrm{OR}=12,5)$, história de sibilância anterior $(\mathrm{OR}=7,7)$, desmame precoce $(\mathrm{OR}=2,3)$, uso de bico $(\mathrm{OR}=1,9)$, mãe fumante $(\mathrm{OR}=1,7)$, idade abaixo de seis meses $(\mathrm{OR}=1,7)$ e sexo masculino $(\mathrm{OR}=1,5)$.

\section{DISCUSSÃO}

As doenças respiratórias agudas permanecem como a causa mais freqüente de mortalidade infantil nos países em desenvolvimento.

Apesar de parecer óbvio e simples, a atenção dispensada pelos órgãos públicos na prevenção primária poderia ser mais efetiva, a partir da intervenção sobre os fatores de risco sabidamente relacionados com o processo da doença. Parece oportuno, portanto, a execução de estudos

Tabela 2. Comparação de casos de hospitalização por doença respiratória aguda e controles, em análise bivariada, quanto às características pessoais, antecedentes patológicos e estado vacinal. Pelotas, RS, 1998.

\begin{tabular}{|c|c|c|c|c|c|}
\hline Variável & $\begin{array}{l}\text { Casos } \\
\text { N }(\%)\end{array}$ & $\begin{array}{c}\text { Controles } \\
\mathrm{N}(\%)\end{array}$ & OR & IC 95\% & $\mathrm{p}$ \\
\hline \multicolumn{6}{|l|}{ Freqüência à creche } \\
\hline Não & $596(95,4)$ & $143(93,5)$ & 1,0 & & \\
\hline Sim & $29(4,6)$ & $10(6,5)$ & 0,7 & 0,$3 ; 1,5$ & 0,4 \\
\hline \multicolumn{6}{|l|}{ Uso de bico } \\
\hline Não & $74(11,8)$ & $33(22,1)$ & 1,0 & & \\
\hline Sim & $551(88,2)$ & $116(77,9)$ & 2,1 & 1,$3 ; 3,3$ & 0,01 \\
\hline \multicolumn{6}{|l|}{ Hospitalização prévia } \\
\hline Nunca & $469(75,2)$ & $128(85,9)$ & 1,0 & & \\
\hline 1 vez ou mais & $155(24,9)$ & $21(14,1)$ & 2,0 & 1,$2 ; 3,3$ & $<0,01$ \\
\hline \multicolumn{6}{|l|}{ Sintomas respiratórios prévios } \\
\hline Chiado no peito & $265(42,5)$ & $56(36,6)$ & 1,3 & 0,$9 ; 1,8$ & 0,2 \\
\hline Chiado com falta de ar & $185(69,8)$ & $11(19,6)$ & 9,8 & 4,$8 ; 20,0$ & $<0,001$ \\
\hline Pneumonia & $59(9,5)$ & $3(2,0)$ & 6,1 & 1,$7 ; 21,9$ & $<0,01$ \\
\hline \multicolumn{6}{|l|}{ Cartão de vacinação } \\
\hline Sim, visto pelo entrevistador & $442(70,7)$ & $123(80,9)$ & 1,0 & & \\
\hline Não visto/perdeu/não tem & $183(29,3)$ & $29(19,1)$ & 1,7 & $(1,1 ; 2,7)$ & 0,01 \\
\hline
\end{tabular}


Tabela 3. Análise multivariada por regressão logística de casos de hospitalização por doença respiratória aguda, segundo variáveis demográficas socioeconômicas, características maternas e antecedentes da criança. Pelotas, RS, 1998.

\begin{tabular}{|c|c|c|c|c|c|}
\hline \multirow[t]{2}{*}{ Nível* } & \multirow[t]{2}{*}{ Variável } & \multicolumn{2}{|c|}{ Análise bruta } & \multicolumn{2}{|c|}{ Análise ajustada } \\
\hline & & OR (IC 95\%) & $\mathrm{p}$ & OR (IC 95\%) & $\mathrm{p}$ \\
\hline \multirow[t]{3}{*}{1} & Sexo & & 0,01 & & 0,02 \\
\hline & Feminino & 1,0 & & 1,0 & \\
\hline & Masculino & $1,5(1,1 ; 2,2)$ & & $1,5(1,1 ; 2,2)$ & \\
\hline \multirow[t]{3}{*}{1} & Idade da criança & & $<0,01$ & & $<0,01$ \\
\hline & 6 a 12 meses & 1,0 & & 1,0 & \\
\hline & 0 a 6 meses & $1,7(1,2 ; 2,5)$ & & $1,7(1,2 ; 2,5)$ & \\
\hline \multirow[t]{5}{*}{2} & Renda familiar (SM) & & $<0,01$ & & 0,03 \\
\hline & Mais de 6,0 & 1,0 & & 1,0 & \\
\hline & 3,1 a 6,0 & $1,5(0,8 ; 2,8)$ & & $1,3(0,6 ; 2,5)$ & \\
\hline & 1,1 a 3,0 & $2,7(1,5 ; 4,9)$ & & $2,0(1,0 ; 3,9)$ & \\
\hline & Até 1,0 & $5,2(2,7 ; 9,9)$ & & $3,0(1,4 ; 6,1)$ & \\
\hline \multirow[t]{5}{*}{2} & Escolaridade materna (anos) & & $<0,001$ & & $<0,001$ \\
\hline & $\geq 9$ & 1,0 & & 1,0 & \\
\hline & 5 a 8 & $4,0(2,6 ; 6,2)$ & & $3,3(2,1 ; 5,2)$ & \\
\hline & 1 a 4 & $9,2(5,1 ; 16,6)$ & & $7,6(4,0 ; 14,2)$ & \\
\hline & Sem escolaridade & $16,7(3,8 ; 4,0)$ & & $12,5(2,7 ; 57,2)$ & \\
\hline \multirow[t]{5}{*}{3} & Amamentação (meses) & & $<0,001$ & & $<0,001$ \\
\hline & $\geq 6$ & 1,0 & & 1,0 & \\
\hline & 3,1 a 6 & $1,1(0,6 ; 2,1)$ & & $1,7(0,8 ; 3,4)$ & \\
\hline & 1,1 a 3 & $1,9(1,1 ; 3,1)$ & & $2,3(1,3 ; 4,2)$ & \\
\hline & Até 1 mês & $2,2(1,4 ; 3,4)$ & & $2,3(1,4 ; 3,7)$ & \\
\hline \multirow[t]{3}{*}{4} & Trabalho mãe fora de casa & & $<0,01$ & & 0,3 \\
\hline & Não & 1,0 & & 1,0 & \\
\hline & Sim & $0,5(0,3 ; 0,8)$ & & $0,7(0,4 ; 1,3)$ & \\
\hline \multirow[t]{3}{*}{4} & Uso de bico & & 0,01 & & 0,03 \\
\hline & Não & 1,0 & & 1,0 & \\
\hline & Sim & $2,1(1,3 ; 3,3)$ & & $1,9(1,1 ; 3,3)$ & \\
\hline \multirow[t]{3}{*}{4} & No pessoas dormindo com a criança & & $<0,01$ & & 0,05 \\
\hline & Até 3 & 1,0 & & 1,0 & \\
\hline & Mais de 3 & $2,1(1,3 ; 3,2)$ & & $1,7(1,0 ; 2,7)$ & \\
\hline \multirow[t]{4}{*}{4} & Fumo materno ( $\mathrm{n}^{\circ}$ cigarros/dia) & & $<0,01$ & & 0,02 \\
\hline & Não fuma & 1,0 & & 1,0 & \\
\hline & Até 10 & $1,9(1,3 ; 2,9)$ & & $1,7(1,1 ; 2,6)$ & \\
\hline & Mais de 10 & $5,1(1,6 ; 16,5)$ & & $2,9(0,9 ; 10,3)$ & \\
\hline \multirow[t]{3}{*}{5} & Sintomas respiratórios prévios & & $<0,01$ & & $<0,01$ \\
\hline & Chiado com falta de ar & $9,8(4,8 ; 20,0)$ & & $7,7(3,2 ; 18,6)$ & \\
\hline & Pneumonia & $6,1(1,7 ; 21,9)$ & & $2,5(0,4 ; 14,6)$ & \\
\hline
\end{tabular}

* O efeito de cada variável sobre o desfecho foi ajustado para as variáveis de mesmo nível ou de níveis acima no modelo hierárquico (número anterior).

objetivando reconhecer e avaliar a magnitude desses fatores sobre a DRA, o que poderá contribuir favoravelmente nas condições de saúde da população.

Em relação à nomenclatura DRA, optou-se pela sua utilização em função da dificuldade observada na prática pediátrica de discriminar aquelas condições em que o broncoespasmo é manifestação primária preponderante, ou secundário a quadros infecciosos. Adicionalmente, dentre os objetivos do projeto geral da pesquisa, estava o de reconhecer a importância das condições respirató- 
rias agudas, em termos de morbidade na cidade a partir da incidência de internação pela doença. Tais resultados foram publicados previamente. ${ }^{11}$ Dessa forma, além dos quadros de infecções respiratórias, foram incluídas na análise aquelas crianças em que o diagnóstico do árbitro foi de asma aguda, visto que freqüentemente esse quadro tem como fator desencadeante os processos infecciosos, especialmente os virais.

Ainda relacionado ao diagnóstico dos casos, optou-se pela utilização dos critérios da OMS, que priorizam o diagnóstico e tratamento precoce dos casos graves, com maior risco de morte. ${ }^{5}$ Apesar de reconhecer suas limitações, a escolha foi feita em função de sua larga utilização pelos médicos pediatras. Como um dos objetivos do estudo foi avaliar o grau de concordância diagnóstica entre o árbitro do estudo e o médico pediatra, responsável pelo atendimento ao lactente, foi necessária a utilização de critério amplamente aplicado na prática clínica. ${ }^{11}$ A preponderância do sexo masculino quanto à morbidade por DRA é relatada na literatura, ${ }^{9,10}$ assim como detectada na presente análise. Fatores relacionados ao menor calibre da via aérea entre os meninos são os prováveis responsáveis por esse fenômeno. ${ }^{9,13}$

É bem estabelecido o maior risco de mortalidade por DRA associado a idades menores, ${ }^{13}$ especialmente para infecções virais, como a bronquiolite, sendo fator prognóstico para esta condição. ${ }^{4}$ Condições como imaturidade imunológica e o calibre reduzido da via aérea favorecem a evolução do quadro para formas graves, com disfunção respiratória significativa. São também fatores prováveis responsáveis pela maior incidência e gravidade de condições respiratórias entre as crianças mais jovens. ${ }^{1,3}$

Em relação ao tabagismo materno, observou-se, na análise bruta, um efeito dose-resposta em relação ao risco de hospitalização por DRA e à quantidade de cigarros fumados. Porém, após controle para fatores de confusão, apenas o fumo materno em quantidade de até dez cigarros/dia foi fator de risco para o desfecho. Resultado similar foi observado em estudos ${ }^{13,14}$ que avaliaram a prevalência e as taxas de hospitalização por doença respiratória, embora outros ${ }^{2,18}$ não tenham encontrado associação entre o tabagismo materno e doença respiratória. ${ }^{18}$ Como o desfecho avaliado nesses estudos $^{2,18}$ foi a pneumonia, uma das hipóteses é que a exposição passiva ao tabagismo associa-se, mais marcadamente, a outras formas de doença respiratórias, especialmente àquelas acompanhadas de estados broncoespásticos.

O uso de bico foi fator de risco na análise bruta e na ajustada, determinando uma razão de chance para o desfecho cerca de duas vezes maior. Essa associação não é relatada pela literatura. Num primeiro momento, considerou-se que a interferência do bico sobre o desfecho de interesse estivesse relacionada ao aleitamento materno, visto a existência de evidências científicas que o uso do bico reduz o tempo de amamentação. ${ }^{7,17}$ Porém, mesmo após análise multivariada, em que a interferência da amamentação foi adequadamente controlada, manteve-se o efeito do uso do bico sobre a ocorrência de hospitalizações por DRA. Um dos mecanismos envolvidos nesta associação pode ser a facilidade da respiração oral em detrimento da nasal, impedindo que as fossas nasais realizem suas funções de filtração e umidificação do ar inspirado. Outra hipótese é que a troca de bico, prática comum entre crianças, especialmente as freqüentadoras de creches, facilite a contaminação das secreções respiratórias, propiciando a propagação de microorganismos patogênicos. Essa associação, no entanto, deve merecer investigações futuras para seu melhor entendimento.

Em relação à amamentação, mesmo após ajuste para fatores de confusão, observou-se associação do tipo dose-resposta, com tendência linear significativa a aumento das hospitalizações por DRA à medida que o tempo de amamentação diminuía. Resultado similar foi observado em outros estudos em relação à morbidade por doença respiratória. ${ }^{10,13,15}$ Existe, no entanto, descrição de alguns resultados negativos em relação ao aleitamento materno. ${ }^{3,7}$ Fatores possivelmente relacionados a esses resultados incluem a forma da coleta do dado (variável dicotômica x variável quantitativa), avaliação do aleitamento materno como forma exclusiva de alimentação e o viés da causalidade reversa. ${ }^{17}$ Crianças com DRA, particularmente as mais severas, podem ter descontinuado o aleitamento materno pela gravidade do quadro respiratório. Outro fator a ser avaliado é a interação do aleitamento materno com outras variáveis nutricionais, como o baixo peso ao nascer e a desnutrição.

A história antecedente de sibilância e falta de ar, mesmo após ajuste para fatores de confusão, foi o segundo fator de risco mais importante para hospitalização por DRA em crianças menores de um ano de idade. Tal associação vem sendo encontrada por outros pesquisadores, especialmente em relação as bronquiolites. ${ }^{8}$ Provavelmente, as crianças com estes antecedentes respiratórios são asmáticas, necessitando de identificação e acompanhamento médico para tratamento específico, visando à prevenção de intercorrências respiratórias agudas. A identificação e acompanhamento cuidadoso destas crianças poderão determinar impacto favorável sobre a prevalência de doenças respiratórias agudas nessa faixa etária.

O papel dos fatores socioeconômicos sobre a morbimortalidade das doenças respiratórias na infância é bem descrito na literatura. ${ }^{3,14} \mathrm{~A}$ escolaridade materna apresentou associação mais forte com o desfecho do que a renda familiar. Tal resultado sugere que a escolaridade materna tenha um papel independente de 
outras características socioeconômicas familiares. A maior escolaridade propiciaria um conjunto de ações relacionadas ao cuidado mais adequado da criança e ao conhecimento de medidas preventivas de saúde, as quais reduzem a morbidade por doença respiratória.

\section{REFERÊNCIAS}

1. Albernaz EP, Menezes AM, Cesar JA, Victora CG, Barros FC, Halpern R. Fatores de risco associados à hospitalização por bronquiolite aguda no período pósneonatal. Rev Saude Publica. 2003;37(4):485-93.

2. Aldous MB, Holberg CJ, Wright AL, Martinez FD, Taussig LM. Evaporative cooling and other home factors and lower respiratory tract illness during the first year of life. Group Health Medical Associates. Am J Epidemiol. 1996;143(5):423-30.

3. César JA, Victora CG, Santos IS, Barros FC, Albernaz EP, Oliveira LM, et al. Hospitalização por pneumonia: influência de fatores socioeconômicos e gestacionais em uma coorte de crianças no Sul do Brasil. Rev Saude Publica. 1997;31(1):53-61.

4. Fischer GB, Teper A, Colom AJ. Acute viral bronchiolitis and its sequelae in developing countries. Paediatr Respir Rev. 2002;3(4):298-302.

5. Fiterman J, Chatkin JM, Chatkin M. Epidemiologia das Infecções Respiratórias Agudas (IRAs). In: Silva LCC, Menezes AMB, editores. Epidemiologia das doenças respiratórias. Rio de Janeiro: REVINTER; 2001. p. 90103.

6. Fonseca CB, Grisi S. Bronchiolitis, respiratory syncytial virus, and recurrent wheezing: what is the relationship? Rev Hosp Clin Fac Med Sao Paulo. 2003;58(1):39-48.

7. Frank AL, Taber LH, Glezen WP, Kasel GL, Wells CR, Paredes A. Brest-feeding and respiratory virus infection. Pediatrics. 1982;70(2):239-45.

8. Holberg CJ, Wright AL, Martinez FD, Ray CG, Taussig LM, Lebowitz MD. Risk factors for respiratory syncytial virus-associated lower respiratory illnesses in the first year of life. Am J Epidemiol. 1991;133(11):1135-51.

9. Iwane MK, Edwards KM, Szilagyi PG, Walker FJ, Griffin MR, Weinberg GA, et al. Population-based surveillance for hospitalizations associated with respiratory syncytial virus, and parainfluenza viruses among young children. Pediatrics. 2004;113(6):1758-64.

10. Koch A, Molbak K, Homoe P, Sorensen P, Hjuler T,
Além disso, há a influência e a interação do nível de escolaridade da mãe sobre outras variáveis relacionadas ao risco de doença respiratória, como tabagismo e desmame precoce.

Olesen ME, et al. Risk factors for acute respiratory tract infections in yong Greenlandic children. Am J Epidemiol. 2003; 158(4):374-84.

11. Macedo SEC, Menezes AMB, Post P, Albernaz E, Knorst $M$. Respiratory syncytial virus infection in children under one year of age hospitalized for acute respiratory diseases in Pelotas, RS. J Pneumol. 2003;29(1):4-8.

12. Martinez FD, Wright AL, Holberg CJ, Morgan WJ, Taussig LM. Maternal age as a risk factor for wheezing lower respiratory illnesses in the first year of life. Am J Epidemiol. 1992;136(10):1258-68.

13 Post CL, Victora CG, Valente JG, Leal MdC, Niobey FM, Sabroza PC. P Fatores prognósticos de letalidade hospitalar por diarréia ou pneumonia em menores de um ano de idade. Estudo de caso e controle. Rev Saude Publica. 1992;26(6):369-78.

14. Prietsch SO, Fischer GB, Cesar JA, Fabris AR, Mehanna $\mathrm{H}$, Ferreira $\mathrm{TH}$, et al. Doença aguda das vias aéreas inferiores em menores de cinco anos: influência do ambiente doméstico e do tabagismo materno. J Pediatr (Rio J). 2002;78(5):415-22.

15. Victora CG, Fuchs SC, Flores JAC, Fonseca W, Kirkwood B. Risk factors for pneumonia among children in a Brazilian metropolitan area. Pediatrics. 1994;93(6 Pt 1):977-85.

16. Victora CG, Huttly SR, Fuchs SC, Olinto MT. The role of conceptual frameworks in epidemiological analysis: a hierarchical approach. Int J Epidemiol. 1997;26(1):224-7.

17. Victora CG, Kirkwood BR, Ashworth A, Black RE, Rogers S, Sazawal S, et al. Potential interventions for the prevention of childhood pneumonia in developing countries: improving nutrition. Am J Clin Nutr. 1999;70(3):309-20.

18. Victora CG, Smith PG, Barros FC, Vaughan JP, Fuchs SC. Risk factors for death due to respiratory infections among Brazilian infants. Int J Epidemiol.1989;18(4):918-25. 